УДК 332.05

\title{
ПОДХОД К ОЦЕНКЕ СИСТЕМНЫХ РИСКОВ НА ОСНОВЕ ИССЛЕДОВАНИЯ ФАЗОВЫХ ИЗМЕНЕНИЙ ЭКОНОМИЧЕСКОГО ПРОСТРАНСТВА
}

\author{
Каргинова В.В., Толстогузов О.В.
}

Институт экономики Карельского научного иентра Российской академии наук, Петрозаводск, e-mail: vkarginowa@yandex.ru

\begin{abstract}
Происходящая трансформация экономического пространства приводит к неоднородности развития территорий и росту системных рисков. Это, в свою очередь, проявляется в дисфункциях экономических систем и дисбалансах на отраслевых рынках. Следовательно, возникает необходимость понимания причин и направлений трансформации пространства, учёта изменившихся вызовов и угроз национальной безопасности. В статье в рамках мезоэкономического подхода предлагается методология исследования фазовых изменений экономических параметров, в частности энтропии. На основе предложенной методологии произведена оценка системных рисков регионов Северо-Западного федерального округа, даются рекомендации, направленные на совершенствование институционального устройства страны и регионов. Делается вывод о возможности повышения экономической эффективности и снижения существующих системных рисков лишь путём построения нового институционального дизайна управления экономикой.
\end{abstract}

Ключевые слова: экономическая система, локальное равновесие, дисфункция, неоднородность, неопределённость, энтропия, национальная безопасность

\section{APPROACH TO ESTIMATION OF SYSTEM RISKS ON THE BASIS OF THE STUDY OF PHASE CHANGES OF THE ECONOMIC SPACE}

\author{
Karginova V.V., Tolstoguzov O.V. \\ Institute of Economics of the Karelian Research Centre of the Russian Academy of Sciences, \\ Petrozavodsk,e-mail:vkarginowa@yandex.ru
}

\begin{abstract}
The ongoing transformation of the economic space leads to heterogeneity in the development of territories and the growth of system risks. This, in turn, manifests itself in the dysfunctions of economic systems and imbalances in the industry markets. Consequently, there is a need to understand the causes and vectors of the transformation of space, taking into account the changed challenges and threats to national security. In the article within the framework of the meso-economic approach, a methodology for studying the phase changes in economic parameters, in particular, entropy, is proposed. Based on the proposed methodology, an assessment of the systemic risks of the regions of the North-West Federal District was made, recommendations aimed at improving the institutional structure of the country and regions are given. It is concluded that it is possible to increase economic efficiency and reduce existing systemic risks only by building a new institutional design of economic management.
\end{abstract}

Keywords: economic system, local equilibrium, dysfunction, heterogeneity, uncertainty, entropy, national security

Анализ развития регионов России показывает, что структурные изменения, происходящие в период кризиса в экономике, находятся в тесной связи с трансформацией пространства. Её результатом стало возникновение пространственной неравномерности, которая выражается в разной концентрации экономической активности в агломерациях и периферии, в поляризации экономического пространства [1], в возрастании системных рисков, в том числе по выполнению социальных обязательств государства [2]. Неоднородность экономического пространства является одной из главных его особенностей и приводит к неравномерному распределению результатов экономической деятельности.

Термин «системный риск» появился в финансовой среде и обозначает риск, при котором то или иное событие способно вызвать потерю доверия или экономических параметров, сопутствующих росту существенной части финансовой системы, которая, в свою очередь, является настолько значительной, что может оказать неблагоприятное воздействие на реальный сектор экономики [3]. Европейский центральный банк рассматривает системный риск как риск наступления и распространения существенного системного события, негативно влияющего на большое число системообразующих финансовых посредников или рынков [4].

В данной работе под системным риском будем понимать вероятность дисфункции экономической системы при её отклонении от равновесия (фазовых изменениях), возникающем из-за действия экстрактивных рыночных институтов, формирования рынков чемберленского типа и аффилированных стратегий агентов. Высокие системные риски приводят к каскадным процессам на межрегиональных отраслевых рынках и в конечном счете к рецессии экономики периферийных регионов [5]. 
Системный риск должен рассматриваться как фундаментальное свойство экономической региональной системы, которое возникает в результате трансформации экономического пространства. Соответственно, при анализе природы появления и распространения системных рисков, для выявления основных триггеров (переходов к состоянию дисфункции) необходимо исходить из причин и особенностей трансформации и эволюции региональной экономической системы и экономического пространства.

Предметом исследования становятся фундаментальные характеристики как экономической системы, так и экономического пространства. В качестве объекта исследований выступает регион, воспринимаемый не просто как таксон (обладающий статистическими измерениями), а как открытая система, как совокупность отраслевых рынков с границами, обусловленными межрегиональной конкуренцией - причём границы рынков проходят по линиям безразличия, что обеспечивает устойчивость локального равновесия [6]. Под локальным равновесием понимается такое состояние системы, при котором её геометрические и макроскопические величины остаются неизменными. Если же происходят фазовые изменения величин, то система становятся качественно иной.

Исследование опирается на общую методологическую базу экономической теории, конкретно-научную методологию новой институциональной и пространственной экономики с применением соответствующего аналитического инструментария. В частности, используемое математическое описание представляет собой идеализированный объект, определенный механизм наглядности и выступает в качестве инструмента аксиоматизации привлекаемых теоретических представлений об объекте в целях конструктивного средства развертывания семантических и иных теоретических предпосылок. В целях научного обоснования выбора стратегии региона в контексте национальной безопасности осуществляется категорийное сопряжение методологий, в частности понятий экономическая система и экономическое пространство.

На мезоэкономическом уровне существует две трактовки категории экономическое пространство.

Во-первых, экономическое пространство можно рассматривать в качестве пространства взаимодействия, а именно системы координат, введённой для фиксации и упорядочивания изменений, поведения акторов. В данном случае в контексте теории познания пространство не является «вещью».
Во-вторых, под экономическим пространством можно понимать переносчика взаимодействия. Тогда это будет всеобщее свойство объективной реальности, то есть «вещь» в теории познания. Перенос взаимодействия осуществляется при помощи институтов, и одновременно институты выступают и ограничением движения. Само движение проявляется в трансакциях, составляющих вместе с агентами экономическую систему.

В отличие от пространства взаимодействия, в пространстве переноса возмущения - трансакции - могут возникать вследствие наличия не центра концентрации деятельности, а разности потенциалов в отдельных точках.

Использование каждой из приведённых выше трактовок экономического пространства может быть обусловлено характером решаемых задач. Авторы, анализируя неопределённость экономического пространства, будут руководствоваться вторым подходом, так как именно он позволяет учесть полный перечень трансакций: не только технологически обусловленные, но и возникающие случайным образом.

В соответствии с информационным подходом оценка неоднородности экономического пространства может быть осуществлена с помощью показателя энтропии. Его величина количественно характеризует степень неравномерности распределения экономических результатов и вероятность равновесного состояния системы, условий его достижения. В рамках замкнутой системы чем больше энтропия, тем менее упорядочена система и меньше её устойчивость. В отношении рынка - это приближение к совершенному состоянию.

Рассмотрим капитализацию активов как результат действия реальных контрактов и рыночных институтов, регулирующих оборот товаров и финансов на межрегиональных рынках (см. подробнее: [7]). Тогда для расчёта энтропии, которая характеризует экономическое пространство, воспользуемся формулой, основанной на подходе К. Шеннона:

$$
e_{i}=\sum_{j=1}^{n}\left[\frac{S_{i j}}{n \overline{S_{i}}} \ln \left(\frac{n \overline{S_{i}}}{S_{i j}}\right)\right] \text {, }
$$

где $e_{i}$ - энтропия $i$-го региона; $n$ - число секторов экономики; $S_{i j}$ - доля валовой добавленной стоимости в величине активов $j$-го сектора $i$-го региона (внеоборотные и оборотные активы за вычетом просроченной дебиторской задолженности); 
$\overline{S_{i}}-$ средняя потенциальная доля валовой добавленной стоимости в величине активов $i$-го региона.

Потенциальная доля валовой добавленной стоимости в величине активов $j$-го сектора $i$-го региона рассчитывается как

$$
S_{i j}=\max \left\{\frac{\rho_{i j}}{a_{i j}}, \frac{\overline{\rho_{j}}}{\overline{a_{j}}}\right\} \forall i \in M,
$$

где $S_{i j}$ - доля валовой добавленной стоимости в величине активов $j$-го сектора $i$-го региона; $\rho_{i j}-$ валовая добавленная стоимость $j$-го сектора і-го региона; $a_{i j}$ - величина активов $j$-го сектора $i$-го региона; $\overline{\rho_{j}}-$ средняя величина валовой добавленной стоимости $j$-го сектора; $\bar{a}_{j}$ - средняя величина активов $j$-го сектора; $M-$ федеральный округ (макрорегион).
Средняя потенциальная доля валовой добавленной стоимости в величине активов по региону - соответственно

$$
\overline{S_{i}}=\sum_{j=1}^{n} S_{i j},
$$

где $\overline{S_{i}}-$ средняя потенциальная доля валовой добавленной стоимости в величине активов $i$-го региона; $n$ - число секторов; $S_{i j}-$ потенциальная доля валовой добавленной стоимости в величине активов $j$-го сектора $i$-го региона.

Данные энтропии за несколько периодов позволяют исследовать эволюцию в фазовом пространстве. В рамках решаемой задачи последовательность смены состояний системы $X$ (значений её параметров) можно описать следующим образом:

$$
\begin{gathered}
F_{1}: E_{0}\left(X_{0}\right) \rightarrow E_{1}\left(X_{1}\right), \\
F_{2}: E_{1}\left(E_{0}, X_{1}\right) \rightarrow E_{2}\left(X_{2}\right), \\
F_{3}: E_{2}\left(E_{0}, E_{1}, X_{2}\right) \rightarrow E_{3}\left(X_{3}\right), \\
\ldots F_{i}: E_{i-1}\left(E_{o}, \ldots E_{i-2}, E_{i-1}, E_{i}, X_{i-1}\right) \rightarrow E_{i}\left(X_{i}\right), \ldots \\
F_{K}: E_{K-1}\left(E_{o}, \ldots E_{K-2}, E_{K-1}, E_{K}, X_{K-1}\right) \rightarrow E_{K}\left(X_{K}\right),
\end{gathered}
$$

где $F$ - оператор преобразования (изменение параметров состояния системы $X$ ), $\mathrm{E}$ - матрица значений энтропии для разных точек экономического пространства.

Приведенные выражения позволяют производить оценку изменений системы (экономического пространства).

На основании предложенных формул была произведена оценка уровня неопределённости по регионам Российской Федерации. В рамках исследования использовались данные Единой межведомственной информационно-статистической системы, в частности значения валовой добавленной стоимости и величины активов контрагентов.

В таблице представлены отклонения матрицы энтропии по Северо-Западному федеральному округу (разница среднего значения по округу и фактического по региону).

Энтропия характеризует неопределённость экономического развития. Российская экономика неустойчива и зависима от внешних рынков, а характер и особенности трансформации существующих вызовов и угроз приводят к повышению неопределённости и росту системных рисков.

Несмотря на то, что год - достаточно краткосрочный период времени, произошло дальнейшее расхождение параметров по регионам. В Калининградской, Ленинградской и Новгородской областях, а также в Вологодской и Псковской областях, Республике Коми уровень энтропии по сравнению со средним значением округа снизился, что говорит об относительном уменьшении неопределённости в данных регионах. Однако если в первых трёх (Калининградской, Ленинградской и Новгородской областях) уровень энтропии меньше среднего значения по округу, то в оставшихся (Вологодской и Псковской областях, Республике Коми) он и в 2014, и в 2015 г. превышал среднее значение.

Иная динамика наблюдалась в Архангельской и Мурманской областях, городе Санкт-Петербурге и Республике Карелия. В данных регионах произошёл рост уровня энтропии по отношению к среднему в округе. При этом особый интерес представляет Республика Карелия, где в 2014 г. энтропия была меньше среднего по округу, а в 2015 г. стала больше. Это можно объяснить приграничным расположением региона и изменением внешнеэкономических условий (например, введением рядом государств санкций в отношении Российской Федера- 
ции, а следовательно, нарушением производственно-хозяйственных связей, цепочек поставщиков и покупателей).

Трансформация внешних и внутренних условий производственно-хозяйственной деятельности привела к принятию в 2015 г. новой Стратегии национальной безопасности Российской Федерации [12].

Есть понимание, что неконтролируемые локальные риски могут перейти в нерегулируемые системные, которые представляют серьезную угрозу для финансовой стабильности и развития как отдельных региональных экономик, так и национальной системы в целом.

При этом стоит помнить, что в настоящее время межрегиональные отраслевые рынки зачастую имеют олигопольную структуру с вынесенным финансовым центром (как правило, в мегаполис). Соответственно, периферийным компаниям, по сравнению с компаниями центра, остаётся значительно меньше возможностей влиять на обращение товара и формируемые финансовые потоки. В этом случае высокая энтропия увеличивает риски дисфункции экономической системы и дисбалансов отраслевых рынков.

Дисфункции экономической системы находят проявление практически во всех сферах деятельности акторов. Так, в финансовой они приводят к дефициту инвестиций, критическому снижению денежной массы, доходов и, соответственно, расходов и сбережений населения. В сфере инноваций дисфункции выражаются в уменьшении активности и институциональной раз- общенности агентов, а также в цифровой дискриминации. Растут инфраструктурные ограничения, ухудшается проницаемость экономического пространства, обостряющиеся социальные проблемы представляют угрозу общественной стабильности.

Минимальное производство энтропии при возрастающей отдаче делает отраслевые рынки и сектора более устойчивыми, однако при этом возникают риски монопрофильной экономики. Изменение энтропии проявляется в уменьшении или увеличении суммы трансакционных издержек и соответствующем распределении в экономическом пространстве финансового результата деятельности.

С одной стороны, справедливо, что ресурсы территории, в частности природные, при благоприятных условиях - тех, что обеспечивают высокий рыночный потенциал, притягивают экономическую активность. С другой стороны, развитие региональных и межрегиональных отраслевых рынков дифференцированных продуктов, возрастающая отдача также являются объяснением региональной специализации.

При этом важно не только понимать, как именно возникает возрастающая отдача на межрегиональных отраслевых рынках, но и найти её оптимальное распределение. В данном случае первоочередное внимание необходимо уделить факторам, трансформирующим свойства экономического пространства и в конечном счете определяющим движение (трансакции), эффекты масштаба и экстернализации, существующие системные риски.

Значения отклонений матрицы энтропии по Северо-Западному федеральному округу, 2014-2015 гг.*

\begin{tabular}{|l|c|c|}
\hline \multicolumn{1}{|c|}{ Регион } & 2014 г. & 2015 г. \\
\hline Архангельская область & 0,05 & 0,12 \\
\hline Вологодская область & 0,24 & 0,23 \\
\hline г. Санкт-Петербург & $-0,10$ & $-0,09$ \\
\hline Калининградская область & $-0,06$ & $-0,19$ \\
\hline Ленинградская область & $-0,19$ & 0,39 \\
\hline Мурманская область & 0,00 & $-0,33$ \\
\hline Новгородская область & $-0,20$ & 0,22 \\
\hline Псковская область & 0,34 & 0,16 \\
\hline Республика Карелия & $-0,42$ & 0,05 \\
\hline Республика Коми & 0,13 & \\
\hline
\end{tabular}

Пр и м ечан и е. * В рамках произведённых расчётов не были учтены данные по сектору государственного управления и обеспечения военной безопасности ввиду имеющихся особенностей функционирования и сектору домашних хозяйств из-за отсутствия данных об активах. В прочих случаях при отсутствии данных о валовой добавленной стоимости или активах в качестве доли добавленной стоимости в активах использовалась 1.

Источник: рассчитано авторами по данным Единой межведомственной информационностатистической системы [8-11]. 
Осознание причин и факторов трансформации, особенностей производства и распределения результатов деятельности, существующих рисков должно лечь в основу экономической политики, проводимой как на федеральном, так и на региональных уровнях. Необходимо обеспечить введение института мониторинга развития субъектов, а также сопряженность их стратегий. Стратегическое индикативное планирование (государственное программирование) будет эффективно дополнять рыночные принципы. В качестве конкретных институтов, направленных на развитие регионов и реализацию приоритетных направлений, стоит рассматривать различные формы государственно-частного партнерства, государственные и частные финансовые инструменты и прочие меры поддержки резидентов периферии с целью увеличения их инвестиционной и инновационной активности.

Неоднородность (гетерогенность и дивергентность) экономического пространства и, как следствие, системные риски переводят экономическую систему в нестабильное состояние, которое может закончиться её дисфункцией или даже разрушением. При этом в рамках неизменной экономической системы и прежней структуры межрегиональных рынков, с экстрактивными рыночными институтами и инфраструктурными ограничениями, от системного риска нельзя застраховаться, вкладываясь в различные виды активов. Системный риск является нехеджируемым. Поэтому в условиях системных рисков диверсификация и действующая инвестиционная политика являются недейственным методом нейтрализации рисков. Следовательно, избежать убытков в ходе осуществления капиталовложений крайне сложно. Это обстоятельство негативно влияет на инвестиционную привлекательность периферии и не позволяет инвестиционной политике приносить большие дивиденды.

Таким образом, предлагаемый подход к оценке неопределённости и фазовых изменений экономического пространства направлен на формирование нового институционального дизайна государственного управления экономикой и внедрения эффективных рыночных институтов в условиях локального равновесия межрегиональных рынков. Это создаст предпосылки для повышения эффективности государственного планирования и в конечном счёте экономи- ческого роста и снижения рисков периферийной экономики.

Статья выполнена в рамках научно-исследовательской работь «Методология исследования эволюичи северных периферийных регионов и разработка механизмов управления их экономическим развитием» № 0224-2015-0004 и Программы Президиума РАН № I.П13 фундаментальных научных исследований «Пространственное развитие России в XXI веке: природа, обшество и их взаимодействие» № 0224-2015-0005.

\section{Список литературы}

1. Колесников Н.Г., Толстогузов О.В. Структурные изменения экономики Северо-Запада России: пространственный аспект / Н.Г. Колесников, О.В. Толстогузов // Балтийский регион. - 2016. - Т. 8, № 2. - С. 30-47.

2. Толстогузов О.В. Аллокация экономической ренты как антисоциальный фактор // Экономическая наука и социальное государство: сборник материалов Круглого стола в рамках программы «МЭФ-2015» (Москва, 26 марта 2015 г.). - М.: ИСЭПН РАН, 2016. - 165 c.

3. Group of Ten «Report on consolidation in the financial sector», January 2001 [Электронный pecypc]. - Режим доступа: http://www.imf.org/extemal/np/g10/2001/01/eng/pdf/file1. pdf (дата обращения: 25.11.2017).

4. European Central Bank. The concept of systemic risk. Special Feature in the December Financial Stability Report, 2009 [Электронный ресурс]. - Режим доступа: http:// www.ecb.europa.eu/pub/fsr/shared/pdf (дата обращения: 25.11.2017).

5. Толстогузов О.В. Системные риски и лимитирующие факторы экономического роста периферийного региона // Устойчивое развитие: общество и экономика: материалы III Международной научно-практической конференции «Устойчивое развитие: общество и экономика» (СанктПетербург, 20-23 апреля 2016 г.). - СПб.: Скифия-принт, 2016. - С. 399-400.

6. Гранберг А.Г. Основы региональной экономики / А.Г. Гранберг. - М.: ГУВШЭ, 2000. -495 с.

7. Каргинова В.В. Методические подходы к комплексной оценке экономического потенциала региона и уровня его использования / В.В. Каргинова // Экономический анализ: теория и практика. - 2016. - № 8. - С. 86-97.

8. Валовой региональный продукт в основных ценах [Электронный ресурс]. - Режим доступа: https:/www. fedstat.ru/indicator/33379.do (дата обращения: 25.11.2017).

9. Внеоборотные активы по данным бухгалтерской отчетности [Электронный ресурс]. - Режим доступа: https://www.fedstat.ru/indicator/37176.do (дата обращения: 25.11.2017).

10. Оборотные активы организаций [Электронный pecypc]. - Режим доступа: https://www.fedstat.ru/indicator/31482.do (дата обращения: 25.11.2017).

11. Просроченная дебиторская задолженность организаций [Электронный ресурс]. - Режим доступа: https://www. fedstat.ru/indicator/31372.do (дата обращения: 25.11.2017).

12. О Стратегии национальной безопасности Российской Федерации: Указ Президента РФ от 31 декабря 2015 г. № 683 [Электронный ресурс]. - URL: http://base.garant. ru/71296054/\#ixzz4wgw01Sxm (дата обращения: 25.11.2017). 